\title{
Coronal magnetograms of solar active regions
}

\author{
B. I. Ryabov ${ }^{1}$, V. M. Bogod ${ }^{2}$, G. B. Gelfreikh ${ }^{3}$, V. P. Maksimov ${ }^{4}$, \\ F. Drago ${ }^{5}$, B. I. Lubyshev ${ }^{4}$, N. G. Peterova ${ }^{2}$, T. P. Borisevich ${ }^{3}$, \\ and D. A. Bezrukov ${ }^{6}$ \\ ${ }^{1}$ Institute of Astronomy of the University of Latvia, Boulevard Rainis 19 Riga, LV-1586, \\ Latvia email: ryabov@latnet.lv \\ ${ }^{2}$ Special Astrophysical Observatory, Nizhnij Arkhyz, 357147 Karachaevo- Cherkessia, Russia \\ email: peterova@fsao.spb.su \\ ${ }^{3}$ Pulkovo Astronomical Observatory, Pulkovskoe shosse 65, 196140 Sankt-Petersburg, Russia \\ email: btp@gao.spb.ru \\ ${ }^{4}$ Institute of Solar-Terrestrial Physics, Lermontov str.126, 664033 Irkutsk, Russia \\ email: maksimov@iszf.irk.ru \\ ${ }^{5}$ University of Florence, Department of Astronomy and Space Science, Largo Fermi 5, 50125, \\ Florence, Italy email: fdrago@arcetri.astro.it \\ ${ }^{6}$ Ventspils International Radio Astronomy Center, Akademijas laukums 1, Riga LV-1050, \\ Latvia email: magnet155@mail.ru
}

\begin{abstract}
A series of coronal magnetograms, related to the active region NOAA 9068 is derived using the effects of the quasi-transverse (QT-) propagation at microwaves. In the assumption that the product of the electron density $N$ and the scale of magnetic divergence $L_{d}$ is constant and equal to $10^{18} \mathrm{~cm}^{-2}$, the sensitivity of the measurements peaks at $B(G) \approx 180 \lambda^{-4 / 3}(\mathrm{~cm})$, where $\lambda$ is the observational wavelength. The radio maps of the Sun taken with the Solar Siberian Radio Telescope (SSRT) at $\lambda=5.2 \mathrm{~cm}$ were sampled and converted into the $2 \mathrm{D}$ coronal magnetograms supplying absolute values of the total magnetic vector $\vec{B}$ in the range $10-30 \mathrm{G}$. Each coronal magnetogram covers the portion of the microwave source in the active region affected by the QT-propagation conditions in the corona.
\end{abstract}

\section{Introduction}

2D coronal magnetograms can be obtained by analyzing the changes of the degree of circular polarization of a microwave source, which take place in the coronal regions where the magnetic field is nearly transverse to the wave propagation vector (QT-regions). The inferred magnetograms refer to this latter portion of the solar corona. Some recent reviews on the radio measurements of the magnetic field in the QT-regions have been published by Alissandrakis (1999), Gelfreikh (1999), and Ryabov (2004).

If the original degree of circular polarization $\rho_{0}$, is undergoing to the QT effect, the resulting magnetic field $B$ (Gauss) in the QT-region reads:

$$
B \approx 2.05 \times 10^{2} \lambda^{-4 / 3}\left[-\ln \left(\frac{\rho+\rho_{0}}{2 \rho_{0}}\right)\right]^{1 / 3},
$$

where $\rho$ is the observed polarization degree effected by QT-propagation and $\lambda(\mathrm{cm})$ is observational wavelength. Eq. (1) is inferred from Eq.-s (2), (3), given by Zheleznyakov and Zlotnik (1964), under the condition $N L_{d}=10^{18} \mathrm{~cm}^{-2}$ (see Ryabov et al. 2004 for 
a)

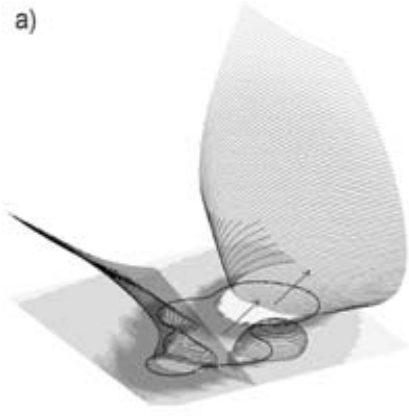

b)
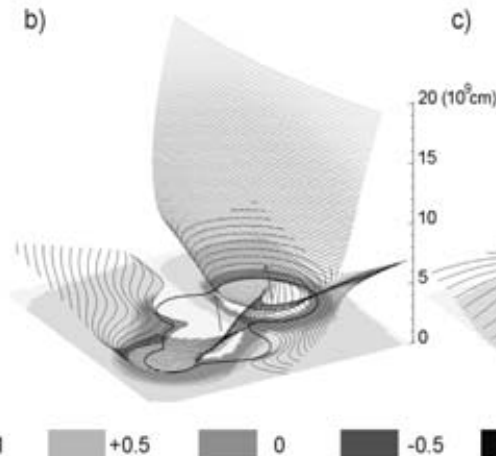

c)
$+0.5$

$-0.5$
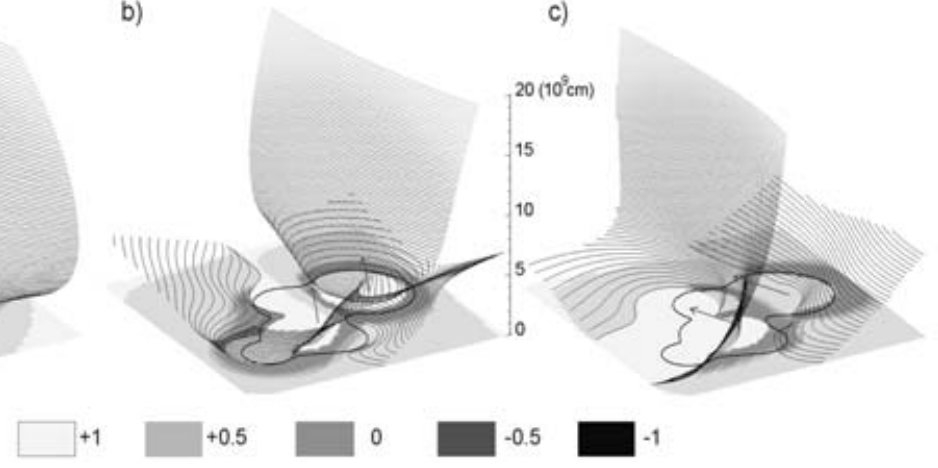

Figure 1. Computed QT-surfaces representing the loci where the rays (arrows) cross the coronal magnetic vectors at the right angle. The coronal field of AR 9068, at the longitudinal displacements: (a) $\theta=-48^{\circ}$, (b) $\theta=-6^{\circ}$, and (c) $\theta=42^{\circ}$ from the central solar meridian, is simulated by the superposition of 5 vertical dipoles. Grayscale images show the modification of circular polarization in the QT region according Eq. (2): the stronger the transformation is, the darker is the area.

justification):

$$
\rho=\rho_{0}\left[2 \exp \left(-2 \delta_{0}\right)-1\right]
$$

where

$$
2 \delta_{0} \approx 1.15 \times 10^{-25} B^{3} N L_{d} \lambda^{4} .
$$

The geometry involved in QT-propagation is illustrated by the imaginary QT-surface, which represents the locus of the points where propagation angle is equal $90^{\circ}$. (Fig. 1 ).

As shown by Eq.-s (2) and (3), the QT effect on circular polarization depends on the physical parameters present in the QT region:

(A) The circular polarization is preserved, $\rho \approx \rho_{0}$, if $\delta_{0} \ll 1$;

(B) The circular polarization is canceled, $\rho=0$, if $\delta_{0} \sim 1$;

(C) The circular polarization is reversed, $\rho=-\rho_{0}$, if $\delta_{0} \gg 1$.

The maximum sensitivity, for the coronal magnetic field measurements $B$, is obtained for $\rho \approx 0.027$. Consequently it is particularly important to know the position of the depolarization line (DL), where $\rho=0$. In terms of the magnetic field, the sensitivity peaks at $B(G) \approx 180 \lambda^{-4 / 3}(\mathrm{~cm})$ (Bogod et al. 2003). One can conclude that, in the centimeter range of wavelength, $\lambda=1-10 \mathrm{~cm}$, this technique provides very reliable measurements of $B$ in the range $180-8 \mathrm{G}$.

\section{Coronal Magnetography}

\subsection{The phenomenon of polarization inversion}

As already mentioned, the inversion of the sense of circular polarization at microwaves provides the basis for the coronal magnetography in the framework of the QT-propagation theory (Ryabov et al. 2004). The usual assumption is that the original circular polarization of the low-lying sunspot-associated sources is supplied by the gyroresonance emission (e.g., Lee et al. 1998; Ryabov et al. 1999; Bogod et al. 2003).

It has been shown by Peterova and Akhmedov (1974), that the sunspot-associated sources present the following common characteristics of the polarization inversion, which merely derived from the geometry of the QT-region separating the opposite magnetic polarities (Fig. 1) and from the physical parameters present in this region, according to Eq. (3): 

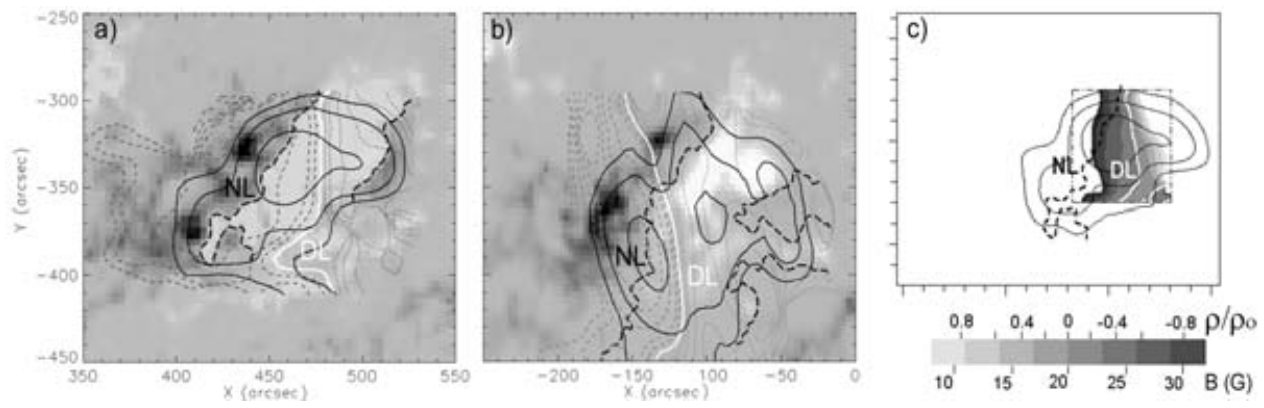

Figure 2. The SSRT $I$ maps (solid contours) and $\rho=V / I$ maps (dashed contours) of the active region 9068 are coaligned with the KPNO magnetograms to illustrate the technique of coronal magnetography. (a) Map of the degree of circular polarization $\rho$ (July 9, 2000). (b) Normalization map $\rho_{0}$ (July 6, 2000, the day of central meridian passage). (c) Two-dimensional coronal magnetogram (grayscale), derived from the normalized map $\rho / \rho_{0}$ according to Eq. (1).

(1) The limbward portion of the microwave source is the first one that inverts its sign of circular polarization in an AR;

(2) The closer an AR is to the solar limb, the shorter is the wavelength required to detect the polarization inversion;

(3) The depolarization line (DL), $\rho=0$, moves toward the western limb while the inversion is observed at a fixed wavelength.

The above characteristics, mostly the wavelength dependence (2), represent therefore a useful diagnostic for the presence of the QT-inversion. There are in fact other causes of polarization inversion, which can be ascribed to a negative temperature gradient in the transition region, as suggested by Zlotnik (1999), or, for flare-productive active regions, by Bogod and Tokhchukova (2003).

In this paper we consider the polarization inversion in the loop-associated sources (see also Bezrukov et al. 2004) assuming that the polarization inversion characteristics for this type of sources are similar to those, mentioned above, for the sunspot-associated sources and that the original sign of polarization corresponds to that of the extraordinary mode in the underlying magnetic field. However, it must be pointed out that some center-tolimb variations in the circular polarization of the loop legs have been noticed during the transit on the disk, therefore this technique can be applied as long as this variation does not sensibly affects $\rho / \rho_{0}$, the quantity assumed to be due to the QT effect only. Moreover a better check of the polarization inversion characteristics for these types of sources as well as an analysis of the relative position of the coronal loop and the QT-region are necessary (Alissandrakis and Preka-Papadema 1984).

\subsection{The technique and the results of coronal magnetography}

In order to get the largest number of informations from Eq. (1) we have used the full range $(-1,+1)$ of the calculated $\rho / \rho_{0}$. The procedure starts with the selection of a radio map, unaffected by the QT-region, which degree of circular polarization is $\rho_{0}(x, y)=V_{0}(x, y) / I_{0}(x, y)$ : a microwave source associated to a bipolar AR can be considered unaffected by the QT-region if it looks like a bipolar microwave source, with the sense of circular polarization corresponding to that of the extraordinary mode (Fig. 2). This map is used for the normalization of a set of AR polarization maps $\rho(x, y)=V(x, y) / I(x, y)$.

The next step is to shift all normalized maps $\rho / \rho_{0}$ to the position of the normalizing map $\rho_{0}$, which is usually near the central meridian. 
Finally, the normalized $\rho / \rho_{0}$ maps are converted into coronal magnetograms using the Eq. (1), in which the product $N L_{d}$ has been assumed constant (Ryabov et al. 1999). The borders of the coronal magnetograms are derived from the reasonable limitation of $\left|\rho / \rho_{0}\right|<1-\sigma=0.95$, where $\sigma$ represents the relative accuracy of the $\rho$ maps. In case of the SSRT observations, $\sigma=0.05$.

The results obtained applying this technique to the AR 9068 are shown in Fig. 2c. AR 9068 was observed by the SSRT from July 3 to July 10, 2000. During its transit on the solar disk the radio source underwent to a polarization inversion in each solar hemisphere, thus supplying two sequences of coronal magnetograms (Bezrukov et al. 2004). The comparison of Fig. 1c and Fig. 2c shows a remarkable similarity between the QT surface and the magnetogram. From this comparison we notice that the field gradient is directed toward the magnetic neutral line, which is located at the bottom of the QT surface. This indicates that the inferred magnetic fields decrease with height as expected. Such a result can give us an idea of the magnetic field structure in the QT-region, but cannot supply any information on its height in the solar corona.

\section{Discussion and Conclusions}

The radio mapping of the Sun with the SSRT at $5.2 \mathrm{~cm}$ and with the NoRH at 1.76 $\mathrm{cm}$ have provided data for 2D coronal magnetography through QT-propagation in the ranges $10-30 \mathrm{G}$ and $50-110 \mathrm{G}$ respectively (Ryabov et al. 2004). Our recent attempt (see Bezrukov et al. 2004) to get a long series of the coronal magnetograms of extended circular polarized sources is encouraging. The inferred magnetograms present the same simple geometry of the corresponding QT-regions, nevertheless, we realized that, in order to have more quantitative results, the loop-associated sources need further investigations. Besides this, one might expect that the characteristics of the polarization inversion for the loop-like sources are not the same as those mentioned for a sunspot-associated source.

\section{Acknowledgements}

This work has been supported by INTAS grants Open Call 2000 00-0181 and 00-0543.

\section{References}

Alissandrakis, C. E. 1998 Proceedings of the Nobeyama Symposium, Kiyosato, Japan, Oct. 27-30 (ed. T. Bastian \& N. Gopalswamy \& K. Shibasaki). NRO Report No.479, 53-58.

Alissandrakis, C.E. \& Preka-Papadema, P. 1984 Astron. Astrophys. 139, 507.

Bezrukov, D. A., Ryabov, B. I., Bogod, V. M., Gelfreikh, G. B., Maksimov, V. P., Drago, F., Lubyshev, B. I., Peterova, N. G., \& Borisevich, T. P. to be published 2004 Baltic Astronomy.

Bogod, V. M., Gelfreikh, G. B., Drago, F. Ch., Maksimov, V. P., Nindos, A., Kaltman, T. I., Ryabov, B. I.,Tokhchukova, S. Kh. 2003 ASTROPAGE astro-ph/03009444 http://lanl.arxiv.org/abs/astro-ph/?astro-ph

Bogod, V. M. \& Tokhchukova, S. Kh. 2003 Astronomy Letters 29, 263.

Gelfreikh, G. B. 1998 Proceedings of the Nobeyama Symposium, Kiyosato, Japan, Oct. 27-30 (ed. T. Bastian \& N. Gopalswamy \& K. Shibasaki). NRO Report No.479, 41-51.

Lee, J., White, S. M., Kundu, M. R., Mikic, Z., \& McClymont, A. N. 1998 Solar Physics 180, 193.

Peterova, N. G. \& Akhmedov, Sh. B. 1964 Soviet Astron. 17, 768.

Ryabov, B. I. 2004 Solar and Space Weather Radiophysics, Chapter 7 (ed. D. E. Gary \& C. O. Keller). Kluwer ASSL volume, 135-152 2004.

Ryabov, B. I., Maksimov, V. P., Lesovoi, S. V., Shibasaki, K., Nindos, A., \& Pevtsov, A. A. 2004 Solar Physics to be published 2004.

Ryabov, B. I., Pilyeva, N. A., Alissandrakis, C. E., Shibasaki, K., Bogod, V. M., Garaimov, V. I., \& Gelfreikh, G.B. 1999 Solar Physics 185, 157.

Zheleznyakov, V. V. \& Zlotnik, E. Ya. 1964 Soviet Astron. 7, 485.

Zlotnik, E. Ya. 1999 Proc.9th European Meeting on Solar Physics, Florence (ed. A.Wilson), ESA SP-448, 1239. 